\title{
Fractura de cadera en el adulto mayor: la epidemia ignorada en el Perú
}

\section{Hip fracture in elderly: the epidemic ignored in Peru}

Correspondencia Ray Ticse Aguirre ray.ticse@upch.pe

Recibido: 27/01/2016

Arbitrado por pares

Aprobado: 20/04/2016

Citar como:

Palomino L, Ramírez R, Vejarano $J$, Ticse R. Fractura de cadera en el adulto mayor: la epidemia ignorada en el Perú. Acta Med Peru. 2016;33(1):15-20

\author{
Lourdes Palomino ${ }^{1 a}$, Rubén Ramírez ${ }^{1 a}$, Julio Vejarano ${ }^{1,2}$, Ray Ticse ${ }^{1,3}$ \\ 1 Facultad de Medicina Alberto Hurtado, Universidad Peruana Cayetano Heredia. Lima, Perú. \\ 2 Centro de Traumatología y Ortopedia, Hospital Nacional Cayetano Heredia. Lima, Perú. \\ 3 Servicio de Endocrinología, Hospital Nacional Cayetano Heredia. Lima, Perú. \\ a Estudiante de medicina.
}

\section{RESUMEN}

Objetivos: Conocer el tiempo de espera para la instauración del tratamiento quirúrgico en pacientes de un hospital de tercer nivel de atención de Lima-Perú, para luego describir los factores médicos y/o administrativos que se encuentren vinculados al tiempo preoperatorio prolongado y describir las complicaciones más frecuentes durante ese tiempo. Materiales y Métodos: Estudio de tipo descriptivo - retrospectivo durante el primer semestre del 2013. Se midió los tiempos preoperatorios, complicaciones, seguro médico, antecedentes patológicos, características de la cirugía en pacientes hospitalizados con fractura de cadera. Resultados: El $71 \%$ de pacientes fue de sexo femenino. La mediana de edad fue de 66 años. El $70 \%$ de pacientes recibió tratamiento quirúrgico con una mediana del tiempo preoperatorio de 18 días. El $77 \%$ tuvo antecedentes patológicos al ingreso, la mayoría por anemia, hipertensión arterial o diabetes mellitus tipo 2. El 62\% tuvo complicaciones médicas. Los pacientes con tratamiento quirúrgico tuvieron menos porcentaje de complicaciones ( $47 \%$ ) que los que no recibieron tratamiento quirúrgico (94\%), así como menos estancia hospitalaria. La mediana de estancia hospitalaria en los pacientes con tratamiento quirúrgico fue de 26 días y la de los que no recibieron tratamiento quirúrgico fue 41 días. Conclusiones: El tiempo preoperatorio fue mayor a las 2 semanas en la mayoría de los casos, especialmente en los pacientes de mayor edad y usuarios del Seguro Integral de Salud.

Palabras clave:

Fracturas de cadera; Periodo preoperatorio; Adulto mayor (fuente: DeCS BIREME). 


\begin{abstract}
Objectives: Know the waiting time for the establishment of surgical treatment in patients of a third level attention hospital in Lima-Peru for the later description of medical and/or administrative factors that are linked to a prolonged preoperative time and description of the most common complications during that time. Materials and Methods: Descriptive-retrospective study during first semester of 2013. Preoperative times, medical complications, medical assurance, pathological background, surgery characteristics in patients hospitalized for hip fracture were assessed. Results: $71 \%$ of the patients were women. The median of age was of 66 years old. $70 \%$ of the patients received surgical treatment with a preoperative time of 18 days. $76.9 \%$ had a pathological background at the admission, mostly anemia, arterial hypertension and type 2 diabetes mellitus. $62 \%$ had medical complications. Post-surgery patients had a smaller percentage of complications ( $47 \%$ ) than the non-surgery patients $(94 \%)$, as well as a smaller hospitalization rate. The median of hospitalization on surgery patients was of 26 days and on non-surgery patients was of 41 days. Conclusions: The preoperative time was higher than 2 weeks in most of cases, especially on elderly patients and SIS users.
\end{abstract}

Key words:

Hip fractures; Preoperative period; Aged (source: MeSH NLM).

\section{INTRODUCCIÓN}

La fractura de cadera, o también llamada fractura de fémur proximal, sigue en aumento debido a que la población de adultos mayores ha ido creciendo en las últimas décadas. La Organización Mundial de la Salud (OMS) ha estimado que para el año 2050 un total de 6 millones de fractura de cadera ocurrirán en el mundo entero por año, teniendo como consecuencia mayor demanda hospitalaria [1]. El Perú, gran parte de los adultos mayores que presentan una fractura de cadera son atendidos en hospitales del Ministerio de Salud (MINSA).

Actualmente el tratamiento óptimo para la fractura de cadera es quirúrgico, debido a que el manejo conservador conlleva mayor estancia hospitalaria y menor retorno de los pacientes al nivel funcional previo [2-5]. Para el tratamiento quirúrgico se necesitan dispositivos metálicos de fijación de fracturas como la placa DHS (Dinamic Hip Screw) (Figura 1). Según lo recomendado por las Guías de Práctica Clínica (GPC), para que los resultados sean óptimos el tratamiento quirúrgico debe aplicarse lo más pronto posible, idealmente dentro de las de las 36 a 48 horas del evento $[2,3]$.

Existen factores que retrasan el tratamiento, éstos pueden ser de índole médico como las enfermedades sistémicas descompensadas, infecciones extra e intrahospitalarias; o también factores relacionados con la organización del hospital: demora en la realización de los riesgos quirúrgicos y neumológicos, insuficientes salas de operaciones, y finalmente factores administrativos o gerenciales como es la ausencia de planificación anual: los hospitales públicos no cuentan con un stock de implantes, como el DHS para tratar las fracturas de cadera [6-11].

Nuestro estudio busca determinar si en un hospital público de Lima-Perú, las fracturas de cadera están siendo operadas en el tiempo recomendado. Así mismo busca encontrar los factores relacionados a la demora del tratamiento. La importancia de esta evaluación radica en que el tiempo preoperatorio prolongado está asociado a mayores complicaciones médicas y mortalidad.

\section{MATERIALES Y MÉTODOS}

Estudio de tipo descriptivo y retrospectivo, realizado en el Hospital Nacional Cayetano Heredia (HNCH) de Lima-Perú, el cual es un hospital general que pertenece al Ministerio de Salud del Perú (MINSA). La información fue obtenida de las historias clínicas y fue recolectada por un médico general y un médico especialista mediante una ficha de datos.

Ingresaron pacientes mayores de 60 años hospitalizados en el Servicio de Traumatología del $\mathrm{HNCH}$, con el diagnostico de fractura de cadera de cualquier etiología, durante el periodo comprendido entre el 1 de enero del 2013 al 30 de junio del 2013. Se excluyeron las historias de pacientes que fallecieron antes de las 48 horas del internamiento, debido a que no se podría establecer el tiempo preoperatorio $u$ otras variables del estudio. También se excluyeron los casos secundarios a neoplasias óseas.

Se obtuvo los datos de edad, sexo y antecedentes patológicos de la historia clínica de ingreso al Servicio de Traumatología. Los datos síndromes geriátricos (inmovilidad, estreñimiento, polifarmacia, deterioro cognitivo, malnutrición, trastorno del sueño y depresión) previo y posterior al ingreso hospitalario, y el índice de Katz (índice de evaluación de las actividades de la vida diaria, que va de la "A" a la "G") previo a la fractura fueron obtenidos de las evaluaciones geriátricas realizadas durante la estancia hospitalaria. El tiempo de enfermedad (tiempo que transcurre desde el evento traumático hasta el ingreso a emergencia del hospital) y el tiempo de estancia hospitalaria (tiempo que transcurre desde la fecha de ingreso a la fecha de alta) se obtuvieron del formato de epicrisis. El tiempo preoperatorio (tiempo que transcurre desde la fecha de ingreso al hospital hasta la fecha de ingreso a sala de operaciones), y la técnica quirúrgica utilizada, es decir artroplastia o reducción abierta y fijación interna (RAFI), fueron obtenidos de la hoja de reporte operatorio. 


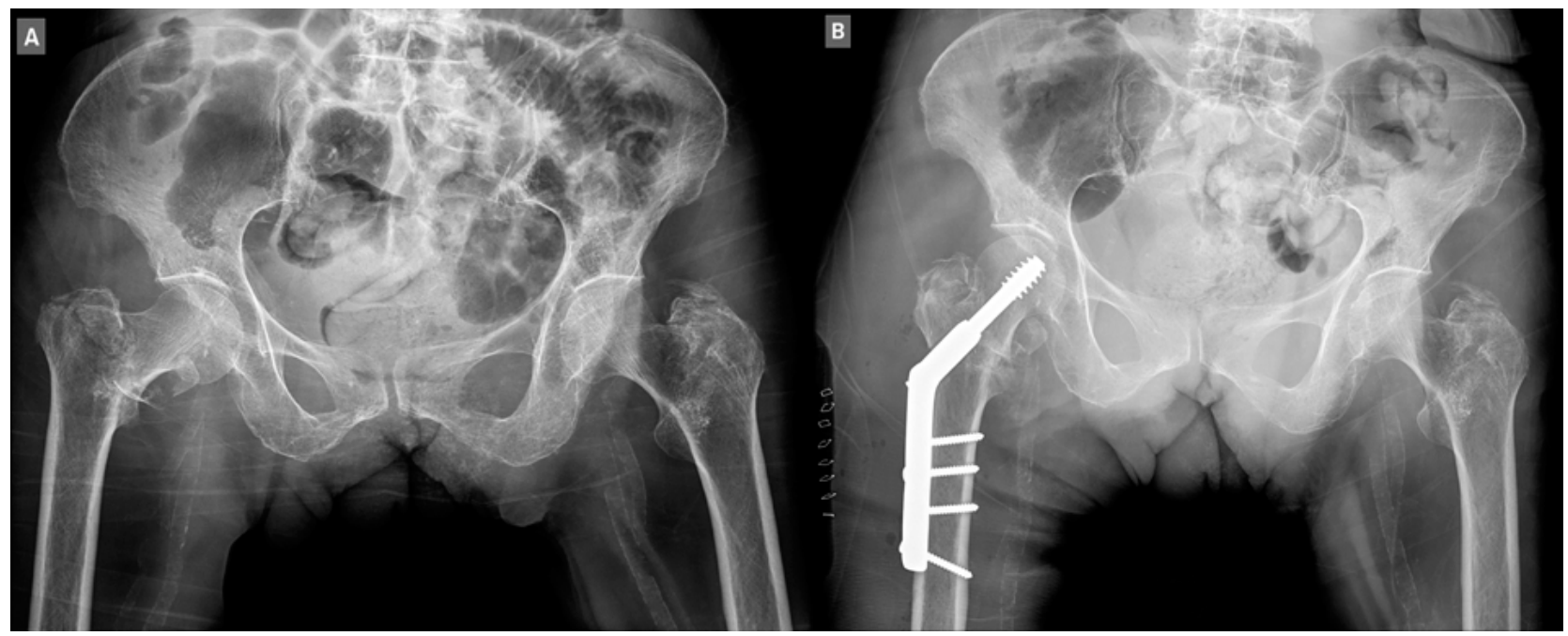

Figura 1.A. Radiografía anteroposterior de pelvis de una paciente de 80 años de edad. Se evidencia una fractura intertrocantérica de la cadera derecha con desplazamiento varo. B. Radiografía control luego de tratamiento quirúrgico. Se ha utilizado un sistema DHS lográndose la restauración de la angulación normal de la cadera derecha.

El tipo de fractura y la clasificación según Müller [12] fueron analizados mediante la observación directa de las placas radiológicas con las que cuenta el Servicio de Traumatología, de manera virtual. Las complicaciones durante el periodo preoperatorio y postoperatorio, así como el tiempo de respuesta de los riesgos cardiológicos y neumológicos, se obtuvieron de las evaluaciones de los servicios de medicina interna, neumología y cardiología. Si el paciente era beneficiario del SIS (Seguro Integral de Salud) o no, fue obtenido de la ficha de ingreso del $\mathrm{HNCH}$, finalmente el tiempo de demora en la compra del material quirúrgico fue obtenido de las evoluciones médicas diarias que realiza el Servicio de Traumatología.

\section{Análisis Estadístico}

Las variables categóricas fueron presentadas en valores de frecuencia. Las variables numéricas se presentaron como medianas y rango intercuartílico (RIQ) por no tener distribución normal. Se compararon los grupos de pacientes que recibieron tratamiento quirúrgico y los que no mediante el uso de la prueba exacta de Fisher para variables categóricas; y la suma de rangos de Wilcoxon para las variables numéricas. El análisis estadístico se realizó con el software estadístico STATA 11. El valor de $p<0,05$ fue considerado como estadísticamente significativo.

\section{Consideraciones Éticas}

El proyecto fue revisado y aprobado el Comité de Ética Institucional de la Universidad Peruana Cayetano Heredia.

\section{RESULTADOS}

Ingresaron al Servicio de Traumatología del $\mathrm{HNCH} 79$ pacientes con fractura de cadera, de los cuales 52 cumplían con los criterios de inclusión (Tabla 1). La mediana de edad fue de 66 años (RIQ: 65 $-81,5)$ y el $71 \%$ de fueron de sexo femenino. Según la clasificación de AO Müller, el $78,7 \%$ de fracturas fue de tipo extracapsular
(31-A) y el 21,3\% fue de tipo intracapsular (31-B) [12]. Fueron 36 (70\%) pacientes los que tuvieron tratamiento quirúrgico, en el $50 \%$ se realizó artroplastia parcial de cadera con prótesis tipo Thompson y en el $50 \%$ restante se realizó RAFI.

Tabla 1. Pacientes con fractura de cadera ingresados en el estudio.

\begin{tabular}{lcl}
\multicolumn{1}{c}{ Variables } & n & $(\%)$ \\
Mujer & 37 & $(71)$ \\
Edad (años)* & 66 & $(65-81)$ \\
Estancia hospitalaria (días)* & 30 & $(20-44)$ \\
Seguro Integral de Salud & 38 & $(73)$ \\
Tipo de fractura** & & \\
$\quad$ Intertrocantérica & 33 & $(64)$ \\
$\quad$ Subtrocantérica & 8 & $(15)$ \\
Transcervical & 9 & $(17)$ \\
$\quad$ Subcapital & 2 & $(4)$ \\
Operados & 36 & $(70)$ \\
Técnica Operatoria & & \\
RAFI & 18 & $(50)$ \\
Prótesis Thompson & 18 & $(50)$ \\
Sin antecedentes patológicos & 14 & $(27)$ \\
Antecedentes patológicos & & \\
Anemia & 28 & $(54)$ \\
HTA & 22 & $(42)$ \\
DBM tipo 2 & 11 & $(21)$ \\
Parkinson & 3 & $(6)$ \\
Cáncer de próstata & 2 & $(4)$ \\
Otras patologías*** & 7 & $(14)$ \\
\hline
\end{tabular}

* Mediana, rango intercuartílico

** Según clasificación de Müller

*** Un caso cada uno: asma, cirrosis, secuela de poliomielitis, hipotiroidismo, enfermedad renal crónica, fibrilación auricular, enfermedad pulmonar intersticial difusa.

RAFI: Reducción abierta y fijación interna; SIS: Seguro Integral de Salud; HTA: Hipertensión arterial; DBM tipo 2: Diabetes mellitus tipo 2. 
Tabla 2. Distribución de pacientes según el tratamiento instaurado.

\begin{tabular}{|c|c|c|c|c|c|}
\hline \multirow{3}{*}{ Variables } & \multicolumn{4}{|c|}{ Tratamiento quirúrgico } & \multirow{3}{*}{ Valor $p$} \\
\hline & \multicolumn{2}{|c|}{$\mathrm{Si}(\mathrm{n}=36)$} & \multicolumn{2}{|c|}{ No $(n=16)$} & \\
\hline & Mediana & (RIQ) & Mediana & (RIQ) & \\
\hline Edad (años) & 66 & $(63-78)$ & 77 & $66-83$ & 0,024 \\
\hline \multirow[t]{2}{*}{ Estancia hospitalaria (días) } & 26 & $(18-36)$ & 41 & $34-54$ & 0,001 \\
\hline & $n$ & $(\%)$ & $\mathrm{n}$ & $(\%)$ & \\
\hline Complicaciones & 17 & (47) & 15 & (94) & 0,002 \\
\hline \multicolumn{6}{|l|}{ Tipo de complicaciones } \\
\hline $\mathrm{NIH}$ & 1 & (3) & 8 & (50) & \\
\hline ITU & 5 & (14) & 1 & (6) & \\
\hline Ulcera de presión & 2 & (6) & 7 & (44) & \\
\hline Deterioro cognitivo & 1 & (3) & 4 & $(25)$ & \\
\hline
\end{tabular}

NIH: Neumonía intrahospitalaria; ITU: Infección del tracto urinario; RIQ: Rango intercuartílico

El $77 \%$ de los pacientes presentó antecedentes patológicos al ingreso. Las más frecuentes fueron anemia ( $85 \%$ anemia leve), hipertensión arterial (HTA) y diabetes mellitus tipo 2 (DM-2) (Tabla 1). Cuatro pacientes tuvieron algún tipo de descompensación de la enfermedad de fondo, 3 por no tener tratamiento regular para DM-2 y uno por azoemia. Sólo el paciente con Azoemia no recibió tratamiento quirúrgico.

Los pacientes que no tuvieron tratamiento quirúrgico mayor mediana de edad y estancia hospitalaria prolongada. A todos los pacientes que ingresaron al servicio de traumatología se les realizo riesgo cardiológico y neumológico. El tiempo de respuesta del riego cardiológico tuvo una mediana de 7 días (RIQ: 3-12) y el tiempo de respuesta del riesgo neumológico tuvo una mediana de 13 días (RIQ: 8-18). (Tabla 2).

De los 36 pacientes tratados quirúrgicamente, el $47 \%$ tuvieron complicaciones y de los 16 pacientes sin tratamiento quirúrgico, el 94\% también las tuvieron, como se evidencia en la Tabla 2 en mayor detalle. En los pacientes con tratamiento quirúrgico la mediana del tiempo preoperatorio fue de 18 días (RIQ: 13-23) y la mediana de estancia hospitalaria fue de 26 días (RIQ: 18-36).

Los pacientes que recibieron tratamiento quirúrgico durante las 2 primeras semanas tuvieron una mediana de edad menor a los pacientes que recibieron tratamiento quirúrgico después de las 2 semanas, con menor porcentaje de complicaciones médicas. El riesgo neumológico fue mayor en los pacientes con mayor tiempo preoperatorio. Los pacientes que demoraron más en la compra de materiales fueron los que entraron a sala de operaciones después de la segunda semana. (Tabla 3).

Los pacientes con SIS tuvieron una mediana de tiempo en la compra de materiales de 17 días (RIQ: 12-23) que fue significativamente mayor a la de los pacientes sin SIS, que tuvieron una mediana de tiempo de 9 días (RIQ: 7-12), $(p=0,007)$.

Tabla 3. Variables de los pacientes que recibieron tratamiento quirúrgico según el tiempo preoperatorio.

\begin{tabular}{|c|c|c|c|c|c|}
\hline \multirow{2}{*}{ Tiempos preoperatorios } & \multirow{2}{*}{$\begin{array}{c}1^{a} \text { semana }(n=1) \\
\text { Mediana }\end{array}$} & \multicolumn{2}{|c|}{$\begin{array}{c}2^{a} \text { semana } \\
(n=14)\end{array}$} & \multicolumn{2}{|c|}{$\begin{array}{c}>2^{a} \text { semana } \\
(n=21)\end{array}$} \\
\hline & & Mediana & (RIQ) & Mediana & (RIQ) \\
\hline Edad (años) & 61 & 65 & $(62-67)$ & 70 & $(66-81)$ \\
\hline Estancia hospitalaria (días) & 12 & 18 & $(15-19)$ & 32 & $(26-43)$ \\
\hline Riesgo quirúrgico (días) & 0 & 7 & $(5-8)$ & 8 & $(3-18)$ \\
\hline Riesgo neumológico (días) & 2 & 8 & $(7-10)$ & 16 & $(13-26)$ \\
\hline Compra de material (días) & 5 & 8 & $(7-10)$ & 18 & $(15-26)$ \\
\hline Complicaciones & $0 / 1$ & $4 / 14$ & (29) & $13 / 21$ & $(62)$ \\
\hline
\end{tabular}

RIQ: Rango intercuartílico 


\section{DISCUSIÓN}

Las guías traumatológicas sugieren que el tratamiento quirúrgico es el de elección y debe ser instaurado dentro de las primeras 48 horas, sin embargo en el hospital de estudio ningún paciente recibió tratamiento quirúrgico precozmente. Cuando revisamos la bibliografía nacional y extranjera encontramos cifras de tiempos preoperatorios que generalmente son menores que las nuestras.

En un estudio realizado en el Hospital Nacional Guillermo Almenara Irigoyen de EsSalud porVillon J. et al. se encontró que sólo el 5,3\% recibió tratamiento quirúrgico dentro de las primeras 48 horas y el $32,7 \%$ lo hizo dentro de la primera semana. Un $13,7 \%$ tardó hasta un mes y finalmente un $4,1 \%$ lo hizo después del mes [13]. Pérez-Ochagavía reportó que la mediana de tiempo preoperatoria era de 5 días (rango de o a 18 días) en un hospital de Salamanca [14]. Amigo et al, encontraron una mediana de tiempo preoperatorio de 41,8 horas en un hospital universitario de Cuba [15]. Esta demora en el pre quirúrgico es un factor de riesgo para presentar complicaciones médicas y mayor tiempo de hospitalización [16].

En cuanto al tiempo preoperatorio prolongado, nuestro estudio encontró una diferencia significativa en cuanto a la edad de los pacientes. Los adultos mayores se operaban después de mayor tiempo y muchos no recibían tratamiento quirúrgico. Sin embargo otros estudios reportaron resultados diferentes. En la publicación de Pérez-Ochagavía et al. no se halló diferencia significativa con respecto a la edad y el tiempo preoperatorio [14]. Amigo $P$ et al., encontró que las únicas variables que prolongaban el tiempo preoperatorio de forma significativa fueron los antecedentes patológicos como hipertensión arterial y diabetes mellitus tipo 2 [17]. A diferencia de nuestro estudio, en el que la mayoría de pacientes no presentaron complicaciones a raíz de los antecedentes patológicos.

El tiempo preoperatorio se prolonga en muchos casos por la necesidad de realizar procedimientos y evaluaciones prequirúrgicas. En el $\mathrm{HNCH}$ todos los pacientes cuentan con una evaluación de riesgo quirúrgico al ingreso por Emergencia, el cual se realiza dentro de las primeras 24 horas, sin embargo a todos pacientes le solicitan una evaluación cardiológica adicional como requisito preoperatorio, la cual no se realiza a la brevedad debido a la sobredemanda de interconsultas que tiene la especialidad de cardiología [11]. Es necesario que se considere a la fractura de cadera como una urgencia médica de modo que se implementos los procesos que permitan una evaluación temprana.

Otro de los factores que se ven relacionados a la prolongación en el tiempo que demanda el procedimiento para obtener el riesgo neumológico. En general, se recomienda que de manera regular la evaluación neumológica clínica la realice el médico anestesiólogo y sólo se solicite la evaluación por el médico neumólogo en casos de descompensación de enfermedades pulmonares crónicas o infecciones agudas [18]. En nuestro medio, además de la evaluación por el médico neumólogo a todos los pacientes adultos mayores se les solicita análisis de gases arteriales, radiografía de tórax y espirometría. Sobre lo último, nosotros no estamos de acuerdo en su aplicación de manera regular en los hospitales del MINSA por las siguientes razones: primero porque no está disponible en los servicios de emergencia; segundo, porque la espirometría preoperatoria de rutina juega un pobre o ningún papel en los pacientes con fractura de cadera. Tercero, el valor predictivo de la espirometría para evaluar posibles complicaciones postquirúrgicas no es mejor que los hallazgos clínicos, más bien incrementa el tiempo preoperatorio [6].

Según la ley de aseguramiento universal, el SIS cubre prestaciones de salud en base al Plan Esencial de Aseguramiento en Salud (PEAS), en el que está incluida la cobertura total de las condiciones agudas como las fracturas. La mayoría de pacientes están afiliados a dicho seguro, por lo que los materiales pre quirúrgicos son solicitados mediante ese medio, sin embargo el tiempo de demora en la compra de materiales es prolongado, por lo que se hace indispensable contar con un stock de los insumos requeridos para las cirugías de cadera y no recién iniciar el proceso con cada evento. El tiempo que demandó la compra de insumos prolongo de manera considerable al tiempo preoperatorio, lo que afecta de manera negativa la recuperación funcional del paciente, genera menos carga de enfermedad, alivia los costos a la familia y los servicios de salud. Muchos de los patrones de fractura de cadera pueden resolverse con implantes de fijación interna como el DHS, este implante es asequible en nuestro medio. Si el SIS planifica y concreta compras anuales de este tipo de implante o similares, aseguraría su disponibilidad oportuna y también bajaría su costo de adquisición.

El excesivo tiempo preoperatorio estuvo asociado a complicaciones médicas, en su mayoría por neumonía, infecciones urinarias y ulceras de presión. A partir de la segunda semana de hospitalización el porcentaje de pacientes que presentó dichas complicaciones aumentó significativamente, resultados similares obtuvieron Rodriguez et al., quienes evaluaron a un grupo de adultos mayores que ingresaron a sala de operaciones por fractura de cadera y encontraron que las complicaciones fueron más frecuentes en los pacientes que demoraron más de una semana. Las tasas de aparición de úlceras por presión, infecciones urinarias, la trombosis venosa profunda y neumonía fueron mayores en estos pacientes [10]. Villon et al. encontraron que el $89,1 \%$ de sus pacientes en el HNGAI-EsSalud tuvieron complicaciones durante el periodo pre operatorio. Las más frecuentes fueron delirium (50\%), infecciones urinarias $(25,6 \%)$ y neumonía intrahospitalaria $(16,7 \%)$. Sin embargo, no mencionan si la relación con la demora en el tiempo preoperatorio fue significativa estadísticamente [13]. Pérez-Ochagavía refiere que en su estudio se obtuvieron diferencias estadísticamente significativas al relacionar la estancia media hospitalaria y la existencia o número de complicaciones [14].

El tratamiento quirúrgico en el hospital de estudio fue instaurado en $70 \%$ de los pacientes, el cual es un porcentaje bajo en comparación con otros hospitales. Villon et al. refieren que en el HNGAI-EsSalud se realizó tratamiento quirúrgico al 95,1\% 
de pacientes [13]. Las GPC de fractura de cadera refiere que en la actualidad existe el consenso generalizado de que, con la excepción de pacientes de edad muy avanzada con un estado muy crítico, y aquellos que ya no caminan, el tratamiento de elección de este tipo de fracturas será siempre el quirúrgico $[3,19]$.

Entre las limitaciones del estudio consideramos al limitado número de muestra, el sólo considerar la experiencia de un hospital, falta de reporte de los casos de no incluidos en la presente muestra. Tampoco se realizó el seguimiento posoperatorio para determinar el resultado final en cuanto a recuperación funcional, rehospitalizaciones y mortalidad.

Como conclusión tenemos que ningún paciente fue intervenido en el tiempo recomendado por las GPC, el tiempo preoperatorio fue más de dos semanas en la mayoría de los casos, especialmente en pacientes adultos mayores y en usuarios del SIS. La estancia hospitalaria es mayor en los pacientes sin tratamiento quirúrgico y tuvieron mayor frecuencia de complicaciones médicas.

Recomendamos que el ministerio de salud implemente un sistema de compras y abastecimiento de insumos requeridos para que la cirugía de cadera se realice en el tiempo recomendado por las GPC, con el fin de retornar al paciente a su nivel funcional previo a la fractura y disminuir la alta frecuencia de complicaciones médicas ocurridas por prolongar el tiempo preoperatorio. Se deben optimizar los procedimientos para el ingreso a sala de operaciones, estos deben seleccionarse según el tipo de paciente.

Conflictos de interés: Los autores declararan no tener conflictos de interés con la publicación de este artículo.

Fuente de financiamiento: Autofinanciado

\section{REFERENCIAS BIBLIOGRÁFICAS}

1. World Health Organization (WHO). Prevention and management of osteoporosis, EB11413, 2004. Geneva: WHO; 2004.

2. Scottish Intercollegiate Guidelines Network. Prevention and management of hip fracture in older people: a National Clinical Guideline. Edinburgh, UK: Scottish Intercollegiate Guidelines Network; 2002.

3. Avellana J, Ferrández L. Guía de la buena práctica clínica en geriatría: anciano afecto de fractura de cadera. Madrid: Sociedad Española de Geriatría y Gerontología, Sociedad Española de Cirugía Ortopédica y Traumatológica, Elsevier Doyma; 2007.
4. Muñoz $G$, Lavanderos $F$, Vilches $A$, Delgado $M$, Cárcamo $H_{\text {, }}$

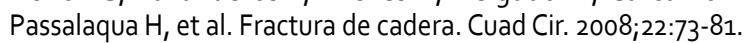

5. Siegmeth AW, Gurusamy K, Parker MJ. Delay to surgery prolongs hospital stay in patients with fractures of the proximal femur. J Bone Joint Surg Br. 2005;87(8):1123-6.

6. Qaseem A, Snow V, Fitterman N, Hornbake E, Lawrence V, Smetana $G$, et al. Risk assessment for and strategies to reduce perioperative pulmonary complications for patients undergoing non cardiothoracic surgery: a guideline from the American College of Physicians. Ann Intern Med. 2006;144(8):575-80.

7. Siu CW, Sun NC, Lau TW, Yiu KH, Leung F, Tse HF. Preoperative cardiac risk assessment in geriatric patients with hip fractures: an orthopedic surgeons' perspective. Osteoporos Int. 2010;21(Suppl 4):S587-91.

8. Hay D, Parker MJ. Hip fracture in the immobile patient. J Bone Joint Surg Br. 2003;85(7):1037-9.

9. McLaughl M, Orosz G, Magaziner J, Hannan E, McGinn T, Morrison R, et al. Preoperative status and risk of complications in patients with hip fracture. J Gen Intern Med. 2006;21(3):21925 .

10. Rodriguez $P$, Adarraga $D$, Carpintero P. Effects of delayed hip fracture surgery on mortality and morbidity in elderly patients. Clin Orthop Relat Res. 2011;469(11):3218-21.

11. Abbas K, Umer M, Askari R. Preoperative cardiac evaluation in proximal femur fractures and its effects on the surgical outcome. Acta Orthop Traumatol Turc. 2012;46(4):250-4.

12. AO Foundation. MüllerAOClassification of Fractures-Long Bones. Switzerland: Grupo AO Trauma de la AO Foundation; 2010.

13. Villon J, Pecho L. Valoración geriátrica integral y aspectos perioperatorios en ancianos hospitalizados con fractura de cadera por caída [Tesis doctoral]. Lima: Facultad de Medicina, Universidad Nacional Mayor de San Marcos; 2003.

14. Pérez-Ochagavía F, Pedro J, Cabo A, Blanco J, Borrego D, Zan J. Estudio epidemiológico de las fracturas proximales del fémur en una población mayor de 69 años durante los años 2000-2001. Rev Esp Cir Ortop Traumatol. 2004;48(2):113-21.

15. Amigo P, Reguera R, Castañeda C, Rodríguez M. Estudio de la estadía hospitalaria en pacientes operados de fractura de cadera. Rev Med Electron. 2005; 27(4):e3.

16. Simunovic N, Devereaux PJ and Bhandari M. Surgery for hip fractures: Does surgical delay affect outcomes? Indian J Orthop. 2011;45(1):27-32.

17. Amigo $P$, Rodríguez M. Complicaciones intrahospitalarias de los pacientes intervenidos de fractura de cadera. Rev Med Electron. 2008;30(5):e1.

18. Lo IL, Siu CW, Tse HF, Lau TW, Leung F, Wong M. Wong. Preoperative pulmonary assessment for patients with hip fracture. Osteoporos Int. 2010;21(Suppl 4):S579-86.

19. American Academy of Orthopaedic Surgeons. Management of hip fractures in the elderly. Evidence- based Clinical Practice Guideline. First Edition. Rosemont, IL: AAOS; 2014. 